DOI: https://doi.org/10.18485/bells.2016.8.7

UDC: 811.111'276.6:004.42

UDC: 371.3:004.7

\author{
Ivana Čorbić* \\ University of Belgrade \\ Faculty of Philology \\ Serbia
}

\title{
PEERS OF THE DIGITAL REALM
}

\begin{abstract}
:
This paper is based on three years of teaching freshmen the basics of argumentative essay writing as part of the coursework for Integrated Skills seminars of Contemporary English language courses at the English Department of the Belgrade University Faculty of Philology. The blended approach to teaching was first suggested as part of this author's JFDP (Junior Faculty Development Program) innovation project based on the experience gained and courses attended at the University of South Carolina, USA in 2012.

Three generations of around 150 students and a team of two teachers worked on process writing both in the new digital environment (provided by the Moodle platform) and in the traditional classroom, combining peer and teacher feedback, and different mediums of communication. In the course of work, the following issues were raised: the issues of digital literacy (what is the minimum and assumed know-how required of digital natives and how comfortable they actually are in a new digital environment), (digital) politeness (especially studentstudent communication online - on the Moodle platform, and face to face), and the nature and quality of feedback (teacher and student perceptions, assumptions and expectations of meaningful and effective feedback). Here politeness can be assumed to include the (development of) strategies to communicate successfully while giving feedback.

This paper presents some of the insights gained using a combination of surveys, interviews, classroom observation/discussion over the three years of work, and an analysis of student submissions.
\end{abstract}

Key words: blended learning, teaching writing, peer feedback, digital literacy, politeness, communication

\footnotetext{
E-mail address: icorbic@hotmail.com
} 


\section{Introduction}

This paper is based on three years (academic years 2012/13, 2013/14, and $2014 / 5$ ) of teaching freshmen the basics of argumentative essay writing as part of the coursework for their Integrated Skills seminars of G1 and G2 Contemporary English language courses at the English Department of the Belgrade University Faculty of Philology. The blended approach to teaching was first suggested as part of this author's JFDP (Junior Faculty Development Program) innovation project based on the experience gained and courses attended at the University of South Carolina in 2012. The original idea was sparked by the course on Technology in Foreign Language teaching, where we learned about a group of post-graduate students who used Internet chat and wikis as means to write their course essays as pair assignments (Elola, Oskoz 2011: 176-180). Given the class sizes at Belgrade University and the intricacies of following individual class contributions and preventing plagiarism, the use of wikis for straightforward collaborative writing was reluctantly abandoned. However, three generations of around 150 students each and a team of two teachers still worked on process writing both in the new digital environment (provided by the Moodle platform) and in the traditional classroom, with combined peer and teacher feedback, and using different mediums of communication. ${ }^{1}$ The students were divided into 6 groups according to their entrance exam results to facilitate best teacher input, and since they were freshmen, the work required initial learner training for pair and group work and work with the Moodle platform. There were further technical constraints such as technical and equipment issues and availability of facilities, which in practice meant that the Moodle (online) work had to be completed by the students at home.

The work on the writing component of G1 and G2 courses focused largely on an introduction to academic writing and training the students to write argumentative essays. In both courses the students had to write individual essays ( 2 in G1 - first semester and 1 in G2 - second semester) but one assignment in each term entailed writing an essay and submitting it for peer review/wiki review by their peers. The peer feedback assignment was part of the final grade for the course and simultaneously an integral part of training for writing the exam essay. The feedback differed in focus

1 Given the number of students and the minimum number of practice essays that needed to be corrected I am grateful to my colleague Milica Prvulović for undergoing the process with me. 
depending on the focus of the course (cohesion-linking words, paragraphs structure, esp. topic sentences for G1, and introductions, thesis statement, and conclusions in G2).

The underlying assumptions for introducing online (wiki) peer feedback were: that students were digital natives, who are comfortable in the digital environment and thus digitally literate (although literacy can always be improved); our belief that students would benefit from reading and reflecting on other people's work (helping them to reflect on their own); that peer review tasks should foster cooperation, improve organisational and communicational skills, and develop critical thinking skills.

The feedback given to students during the process was diverse: each practice essay merited general class feedback (which focused on common errors of structure and organisation), sometimes followed by individual remarks when necessary, while peer feedback essays meant feedback from other students in the wiki comment sections, followed by brief teacher remarks, and final assignments were always marked individually by the teacher with exam-style comments. For the final essay, there was sometimes an additional feedback option following the first draft and before the final submission (Moodle chat option online) or classroom peer feedback. In both these cases students chose their partners themselves within the groups they were assigned to, with the aim to facilitate the task and create/utilise rapport.

Here is an example of the practice essay task with wiki peer feedback:

Choose one of the following topics and write an argumentative essay (introduction, one paragraph for and one against the topic, conclusion):

- English is a world language and so it would be helpful if English words were spelled according to a logical system (e.g. comb, cough and caught should be spelled kome, kof and kort). Discuss.

- Endangered languages should be allowed to die out. Discuss.

- All children should start learning English form the age of six. Discuss.

- There should be a universal language. Discuss.

- The spread of English has a harmful effect on "small" languages like Serbian. Discuss. 
Your essay should be around $\underline{\mathbf{2 5 0}}$ words long. Do not forget to write a plan first. Pay special attention to the structure of your paragraphs: indent the first line, make sure the paragraphs have topic sentences and relevant supporting ideas which you need to connect using appropriate linking words and phrases. Once the time for submitting essays is over, your task is to read the essays of other people from your group and provide meaningful feedback/comment on their work (focusing on the paragraph structure, grammar and vocabulary use and content).

The peer feedback instructions given prior to the assignment included a brief introduction in one of the course books (Zemach, Rumisek 2005: 21-24) on how to give peer feedback, while classroom instructions from the teacher were simply to 'be gentle but truthful' and to exert themselves to be helpful because their partner in the exercise might not return the favour if they did not provide any pertinent comments. This vagueness as to form and structure of the comment stemmed both from the belief that students as digital natives would be comfortable enough working in the online environment but also from a desire to enable them to form their own community of practice should they be inclined to do so.

Following the end of the G2 course and during the G2 orals (exam periods between May and December 2014), generation 2013/4 was given an anonymous questionnaire on their experiences with Moodle/wiki work to complete (participation was completely voluntary) and 58 students responded. The average age of the students was 19-20, where the oldest student who filled in the questionnaire was 22 at the time.

\section{Findings - (Peer) Feedback}

Regarding the main methods for giving feedback (face to face or online -wiki or chat), 22 students (or 38\%) stated that they preferred to give peer feedback face to face, while 34 (59 \%) expressed a preference for doing it online. Among the main reasons given for these respective choices were that it was easier 'to express yourself' and avoid misunderstandings (face to face), or that when feedback was given online there was more time to think and compose the message, and it was thus an easier and more objective method for those who preferred it. As one of the responders explained: 'Not everyone appreciates constructive criticism. I get to word my 
feedback more carefully.' However, to the question about the preferred method of receiving feedback, with the following options: teacher feedback (classroom), teacher feedback (individual), peer (face to face), and peer feedback (wiki/online), most of the students responded that their preferred method was teacher feedback in the classroom. As for the reasons behind their selection, those who expressed a preference for individual teacher feedback stated that it was because they had the full attention of the teacher, and the feedback was more detailed/accurate and impartial. Those with a preference for general class feedback wrote that it was due to the opportunity it provided to see others' work, ask questions and see good examples of possible problems/errors. While some of these preferences are naturally due to the psychological profiles and how extroverted the students might be, there is an underlying belief on the part of the students that, (similar to one of the common complaints while learner training for pair/group in class), only teacher feedback is worth having (corresponding to the belief that speaking with other students is inferior practice compared to having a one-on-one conversation with the teacher). On a Likert scale, the respondents to the questionnaire gave the usefulness of wiki work for learning writing an average mark of 4.49. In general, student comments described it as 'useful', 'practical', 'I enjoy it', and "a good approach to that aspect [of the course]'.

Since the point of the Moodle essay and peer feedback is to provide opportunity to practice writing essays and read and reflect on other people's work and later on one's own, it is essential to offer incentives in the form of percentages of the final mark for each stage of the process to ensure that most students participate in the task. It has also proved to be essential to give some teacher feedback after these sessions, however brief and to the point, not only due to the expectation that the teacher's feedback is more objective and accurate but also to what some observers have labelled 'a tendency to avoid uncertainty' in Serbian students. This feedback after the Moodle peer feedback session also serves as 'proof' that the teacher has in fact read the essays and comments and is not merely a silent observer, which in a way validates the task for the students.

\section{Digital literacy}

Out of the total number of respondents, $67 \%$ percent had had some computer lessons before enrolling at University but only $20 \%$ of the total 
number (30\% of those who had them) said that they had found them useful for working on/with the Moodle platform. At the same time, the students rated their own computer skills as 3.8 on average (on a scale of 1-5). However, despite the initial assumption that digital natives would take to the Moodle like ducks to water, the beginning of the work was littered with glitches of a technical nature (poor internet access at the crucial moment, server issues) and some unexpected enquiries (How do I change the format of a word document? How do I upload the essay to the Moodle? Can you see the essay is from me?). The assumed level of literacy was that the students would be familiar with using Word and a social network (Facebook or any other platform), which should have given them some familiarity with Moodle. However, $86 \%$ of student responders wrote that future generations would benefit from a Moodle orientation session, which was subsequently included in the course for the next generation. Another issue emerged as pertinent: as emails from students encountering difficulties inevitably arrived, some with the essay in the main body of the message instead of an attachment, some with an attachment but no body message, and some even with greetings in the beginning but no names when they signed off, a brief instruction on how to write appropriate formal emails has also been included since.

\section{Digital politeness (Peer to Peer)}

An interesting issue emerged as a result of the questionnaire. Namely, two questions referred directly to politeness, asking whether it was more important to be polite or to be truthful, both face-to-face and online. As expected, $71 \%$ responded that it was more important to be truthful, both face to face and online. There were $12 \%$ of those that felt that it was more important to be polite face to face but truthful online, while $7 \%$ believed that it was more important to be truthful in person but polite online, while only 3 people opted for 'polite' regardless the manner of communication. It seems that online wiki feedback also confers the advantage of making it easier to give critical remarks without facing the person, for the minority who feel that they have to refrain from any criticism face to face. Again the personality profiles of students undoubtedly play a part. Given that students who found themselves in an unfamiliar situation (giving peer feedback), intuitively feel that a certain amount of 'facework' (Brown and 
Levinson 1987) is necessary to mitigate the imposition, it is no surprise the online medium might provide some degree of removal/indirectness to facilitate the task for some students. It was precisely to accommodate the less extrovert students ('Someone can see my work and I'm shy') that the initial 6 big groups of around 25 students were further divided into 4 groups of 5-6 people each. This ensured both that only 5-6 other people can read any one essay and that everyone will get feedback (instead of some essays being read and commented on several times across the group), and hopefully made the task easier for the shy.

Looking at examples of the first wiki peer feedback from a group that initially tested at a good $\mathrm{B}+$ range in the entrance exam, we can notice some general feedback features. These were 24 good students who were further divided into 4 smaller groups. If, as Watts remarks, politic behaviour is based on habit(u)s and experience (Watts 2003: 289, 291), and if, according to Brown and Levinson, politeness is a function of power, distance, and degree of imposition, it is reasonable to assume that these students, faced with an unfamiliar task, chose the format of feedback that would help them deal with the imposition aspect in the best way and be at least somewhat familiar/comfortable for them. Given that the power and distance dimensions were not directly relevant to their communication in a group of peers, although they all knew that the teacher would eventually be reading both the essays and the comments, the degree of imposition (critical remarks leveled at a peer's essay) was probably the most important factor when choosing the format and linguistic means for delivering feedback. The message formats included among others full letter formats ('Dear , ...signed by the student's name), letter salutations (Dear ), and in terms of structure most of them followed the model from their course book to include something positive about the essay, beginning and ending with a compliment. In terms of linguistic devices, most comments included questions, conditionals, and modals, with only one instance of the use of the imperative and passive respectively. Therefore the general characteristic of this group's feedback is that it is indirect. However, there was some 'Netspeak', or typically Internet language, both in terms of spelling and the format: 'All in all $i$ think this essay is a job well-done', 'Oh, and I forgot, I was commenting on an essay by ___, or '@ Milica: your essay is...', which saved the feedback from becoming too formal and was clearly a transfer of existing habits and experience in a new situation. An interesting but not completely unexpected fact (given the Serbian largely prescriptivist 
tradition in language matters) was that as many as one-third of students included comments on the spelling and grammar/vocabulary of the essays even after being instructed to focus on the structure/organisation.

\section{Peer feedback-end of term interviews}

The 2014/5 generation of students had the option of doing their peer feedback in class face to face after they had written the first draft of their final essay for their second term (G2 course). They could record their conversation and send it to the teacher for bonus credit. Out of the 7 interviews submitted with 24 participants (sometimes feedback was given by a group, as a conversation with the author of the essay), a preliminary analysis indicates that in general strategies range from indirect (off record) to bald on record.

Example 1: K: Your vocabulary range is exceptional, and I think, erm, that you managed to fit all the words into the right context. The collocations are cleverly used as well. For example, 'severe impact', 'absolutely talentless', 'artificially created', 'simply adore', 'extremely dangerous', and 'improper behaviour'. Also, everything is perfectly organized.

M: Thanks a lot! I've gone through yours as well, and it lived up to my expectations. You depicted every single aspect of the topic in question, using not only a great range of vocabulary, but also solid grammar constructions. For instance, you've used mixed conditional, passive, the perfect aspect as well.

....However, there are, er, a couple of things that I would change if I were you. First of all, I would shorten the conclusion, and add some, fancy words (...)

K: I've found your criticism very constructive and helpful, thanks. Erm... Concerning your essay, I've also noticed some minuscule details that I would like you to change so that your essay would be flawless, erm ... I think that you should change the topic sentence of the second paragraph, since the way it is now, it resembles the thesis statement ... 
This was the most representative example of indirect strategy, and it may be assumed that the choice of strategy was influenced by the fact that these two students were very aware of the fact that they were recording their conversation and therefore chose to be more formal than expected.

\section{Example 2:}

To begin with, I am pretty satisfied with Ivana's essay. I personally am satisfied with her vocabulary, it's pretty decent. However, there are few spelling and grammar mistakes, but they're not considered to be such an important thing, because those are just minorities, er... as far as the Intro is concerned, her background intro-info is certainly satisfying, she had pretty reasonable arguments, er, and she made it quite clear why procrastination is the biggest problem to her-to exam stress. Also, her thesis statement is, well, it's not really strong but you can't consider it to be weak because it's - it explains a lot, erm. As far as the main paragraph is concerned, erm, her linking <??? $>$ is pretty ok, her topic sentence is...great...

This interview is representative of what the students were expected to have produced, more natural spoken production and less formality with enough information to be constructive.

Example 3:

In terms of essay organization, it's quite good, you clear-clearly spot introduction, two main paragraphs, and the conclusion (...) In terms of the introduction, it's ok. It's nothing special but it's ok, it gives, erm, background on what, er, taking gap year is all about, what does it mean, and when it comes to the thesis statement, I think it is rather weak. (...) When it comes to the mark, I think she should have, she should get a pass mark, but not more than a six [marks awarded are 6-10].

This was the most direct strategy found and the most unexpected interview, given that these two students were perceived to be good friends based on their classroom interaction, where we can see clearly that the student giving feedback prioritized truthfulness and not politeness in a face-to-face interaction and went bold on record. 


\section{Digital politeness - chat}

The same generation of students had the option of giving peer feedback to the first draft of their final essay in the first term (G1) as well but in the form of a Moodle - Internet chat. They still had to work within their groups but they could choose chat partners and the time and the number of sessions on their own. In general, we can conclude that these chat sessions exhibit typical properties of Internet chat of Serbian native speakers (Radić-Bojanić 2007: 38) in terms of their similarity with spoken language, turn-taking features, interruptions, and self-corrections, with the inherent and expected appearance of punctuation errors (leaving out the final punctuation at the end of the sentence, commas, etc.) and typing errors.

It might be interesting to look at an example chat session between two students, one of whom (M) also appeared as one of the speakers in the first example interview:

19:53 M: I definitely enjoyed reading your essay, especially because you tackled every aspect and made your point.

19:54 M: Your vocabulary and grammar are also impressive

19:55 M: However, I think your conclusion is somehow too general

19:57 M: And regarding your spelling, everything is in a perfect order, except you mis-spelled word BEHAVIOUR.

19:57 A: Well, thank you, I'm really glad you liked it

19:58 A: Oh and behaviour wasn't my fault, autocorrect changed it to the American version

19:58 M: :*

19:59 M: Hahahah I completely understand you

This feedback format is probably the most familiar of all those used during the academic year, and although in both cases the student is working on the task with a classmate, this seems to contain the expected and appropriate degree of (in) formality for a conversation with a peer, unlike the interview example (whose format was not unfamiliar except for the demand to 
record the conversation). The use of emoticons and verbalizing laughter is in line with typical Internet chat behaviour. The only surprise regarding chat feedback was that it did not become a favourite among the students, even if it is by nature the least formal and the quickest of all. When offered the option to do the final essay feedback as a chat, most students were against the idea. It seems that the immediacy of the chat abolishes one of the key advantages of giving written feedback online: the opportunity to reflect on the message and take some time to compose it.

\section{Conclusion - Challenges and Reflections}

Even though the online method of giving peer feedback via Moodle wiki tasks has generally proved to be satisfactory, it is regrettably still true that the full potential of the wiki as a collaborative tool cannot be explored in a context where student plagiarism is rampant, without considerable learner training and raising their awareness on the importance of genuine individual contribution. If/when making the decision to try digital peer feedback, technological aspects must be carefully evaluated, given that reliable and fast Internet access and some initial training for both students and teachers are essential, along with good and available technical support. As more and more generations of digital natives come to university it is to be expected that online communication will become more commonplace and in a sense more standardized as some forms of online communication stand the test of time, but for the time being it cannot be expected that digital literacy issues will not crop up even with these new generations. It has been observed in a study email politeness of BU students (Trbojević 2011: 59) that email communication is still perceived as a hybrid medium, and that there are no 'e-politeness' manuals for the academic environment, giving rise to the question of 'whether this type of communication should be taught in an academic setting'. However, given that these issues have persisted in Moodle/wiki work from the first, this might indicate that better training for the new mediums of communication at pre-University levels would be not just common-sense but beneficial. The observed generations of students who are already digital natives (more comfortable typing their essays than writing them by hand, as they still have to do for their final writing exam) seem to be caught in the gap between new technological inventions and the inertia of the educational system. One of the ongoing 
challenges will be to overcome the student prejudice against peer input in both speaking and writing, although studies indicate that Serbian students are not alone in their preference for teacher feedback (Zhang 1995: 209222). Another difficulty which is not inherent to the medium is posed by the (argumentative) essay format itself, given that the essay structure modelled is unfamiliar and contrary to the habits acquired in the students' previous experience (which was usually limited to writing in the native language, and thus more focused on self-expression than argumentation), especially given the relatively small number of words per essay (250-280) which is often perceived as unnecessarily rigid by the students, who on average have been encouraged to write more and in a more elaborate style in primary and secondary school. Although it has been mentioned that 'students don't give good feedback', especially in the beginning of the course when they are still training to both write and evaluate essays, from a teacher's perspective there are definite benefits for critical thinking and reflection. Another important point to mention is that the (wiki) peer feedback puts the focus on the structure of the paragraph/essay and releases the teacher from the obligation to correct every language slip and nuance or spelling errors, which is a significant benefit given the class sizes and the fact that one teacher is responsible for an average number of 75-80 students per year, with a syllabus where writing is just one segment of the material taught in the course. One of the significant advantages of moving peer feedback to the digital realm is also that this frees a portion of class time for other tasks, e.g. more speaking/other skills practice, or vocabulary development, and makes it a more flexible part of the work schedule.

\section{References}

Brown, P. and Levinson, S. (1987) Politeness: Some Universals in Language Usage. Cambridge: Cambridge University Press.

Elola, I and Oskez, A. (2011) Writing Between the Lines: Acquiring the Presentational Mode through Social Tools. In: Arnold, N. and Ducate, L. (eds.), Present and Future Promises of CALL: From Theory and Research to New Directions in Language Teaching, San Marcos, Texas: CALICO $2^{\text {nd }}$ edition, 171-210. 
Ivana Čorbić: Peers of the Digital Realm

Radić-Bojanić, B. (2007) neko za chat? Diskurs elektronskih ćaskaonica na engleskom i srpskom jeziku. Novi Sad: Filozofski fakultet, Novi Sad \&Futura publikacije.

Trbojević-Milošević, I. (2011) Emailing \& Politeness. In: Filološki pregled, Revue de Philologie, Beograd: Filološki fakultet, Vol. 38 (1), 53-61.

Watts, R. (2003) Politeness - Key Topics in Sociolinguistics. New York: Cambridge University Press.

Zemach, D.E and Rumisek, L. A. (2005) Academic Writing from paragraph to essay. Oxford: Macmillan

Zhang, S. (1995) Reexamining the affective advantage of peer feedback in the ESL writing class. Journal of Second Language Writing, Vol. 4(3), 209-222. 